\title{
Posterior Tibial Artery Perforator-Based Adipofascial Flap for Skin Necrosis and Exposed Extensor Tendon after Fixation of Distal Tibiofibular Fracture
}

\author{
Hyunwoo Kyung, Jae-Ik Choi, Seung-Han Song, Sang-Ha Oh \\ Department of Plastic and Reconstructive Surgery, Chungnam National University Hospital, Daejeon, Korea
}

\begin{abstract}
The defect around the distal leg is more problematic due to the paucity of reliable local cutaneous or muscle flaps. The ultimate goal of lower limb reconstruction is to achieve durable soft tissue coverage over stable skeletal repair, and flap choice is made to minimize donor-site morbidity and to maximize lower extremity function. We report our experience with a patient affected by soft tissue defects of the ankle region, who underwent surgical reconstruction with adipofascial flap based on posterior tibial perforator branch. A 60-year-old woman was admitted to our department with a skin necrosis (size $2 \times 2 \mathrm{~cm}$ ) and exposed extensor tendon on the left ankle after fixation of the distal tibiofibular fracture. We performed for reconstruction using posterior tibial artery perforator-based adipofascial flap. After 3 months, flap and donor site remained a well-healed state. The posterior tibial artery perforator-based adipofascial flap, covered with a skin graft, can be one of the suitable methods for reconstruction of the defect of the distal third of leg and provides functional and aesthetic outcome with minimizing donor site morbidity.
\end{abstract}

Keywords: Reconstruction, Posterior tibial artery, Perforator flap

\section{Introduction}

The regions around the distal leg are more problematic due to the paucity of reliable local cutaneous or muscle flaps. The goal of lower limb reconstruction is to achieve durable soft tissue coverage, and flap choice is made to minimize donor-site morbidity and to maximize lower extremity function. When choosing a flap for transfer, considerations must be made regarding flap size, vascular pedicle length and diameter, and the ultimate desired aesthetic outcome.

For defects of the distal third of the lower leg, free flaps are the most commonly performed reconstructive method, but they are relatively complex and require microsurgical expertise and prolonged operating time [1], and free flap reconstruction of the lower extremity has been associated with a failure rate as high as 15 to $20 \%$ [2]. Furthermore, not all patients are willing or healthy enough to undergo free tissue transfer. A locally designed, perforator based, the pedicled flap can provide adequate soft tissue coverage without vascular anastomosis. Earlier perforator flaps were designed with thick subcutaneous tissue around the pedicle that restricted the arc of rotation. Increased understanding of vascular territories, these flaps were designed that is supplied by a single perforating vascular pedicle [1]. The advantage of perforator flaps is that they are safe, reliable, and with minimal donor site morbidity.

The lateral leg is suitable areas for harvesting perforator flaps. The vasculature of the peroneal artery has been well described, and the peroneal artery perforators are \\ Case Report \\ Received: November 13, 2017 \\ Revised: December 14, 2017 \\ Accepted: December 16, 2017

\section{Corresponding author:} \\ Hyunwoo Kyung, M.D.

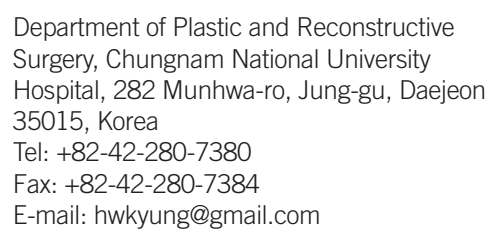

This is an Open Access article distributed under the terms of the Creative Commons Attribution Non-Commercial License (http://creativecommons.org/licenses/by-nc/4.0/) which permits unrestricted non-commercial use, distribution, and reproduction in any medium, provided the original work is properly cited.

(c) 2018 Korean Wound Management Society 
distributed predominately in the lower leg [3]. However, because of trauma, if peroneal artery could not be used, the posterior tibial artery perforator would be the alternative pedicle.

We report our experience with a patient affected by soft tissue defects of the ankle region, who underwent surgical reconstruction with adipofascial flap based on posterior tibial perforator branch.

\section{Case}

A 60-year-old woman was admitted with a skin necrosis (size
$2 \times 2 \mathrm{~cm}$ ) and exposed extensor hallucis longus tendon on the left ankle after fixation of distal tibiofibular fracture (Fig $1 A)$. Because of previous surgery, peroneal artery perforator is not acceptable as flap pedicle. As a result of the peripheral angiogram, the anterior tibial artery was occluded, too. So, we decided to reconstruction using posterior tibial artery perforator-based adipofascial flap. The reason for not having a fasciocutaneous flap is that it has an elevated scar around the donor, which is not appropriate for inclusion of the skin in the flap, and that the primary suture of the donor is also difficult. Preoperatively, the posterior tibial artery perforator is identified and marked on the skin using portable Doppler. The flap
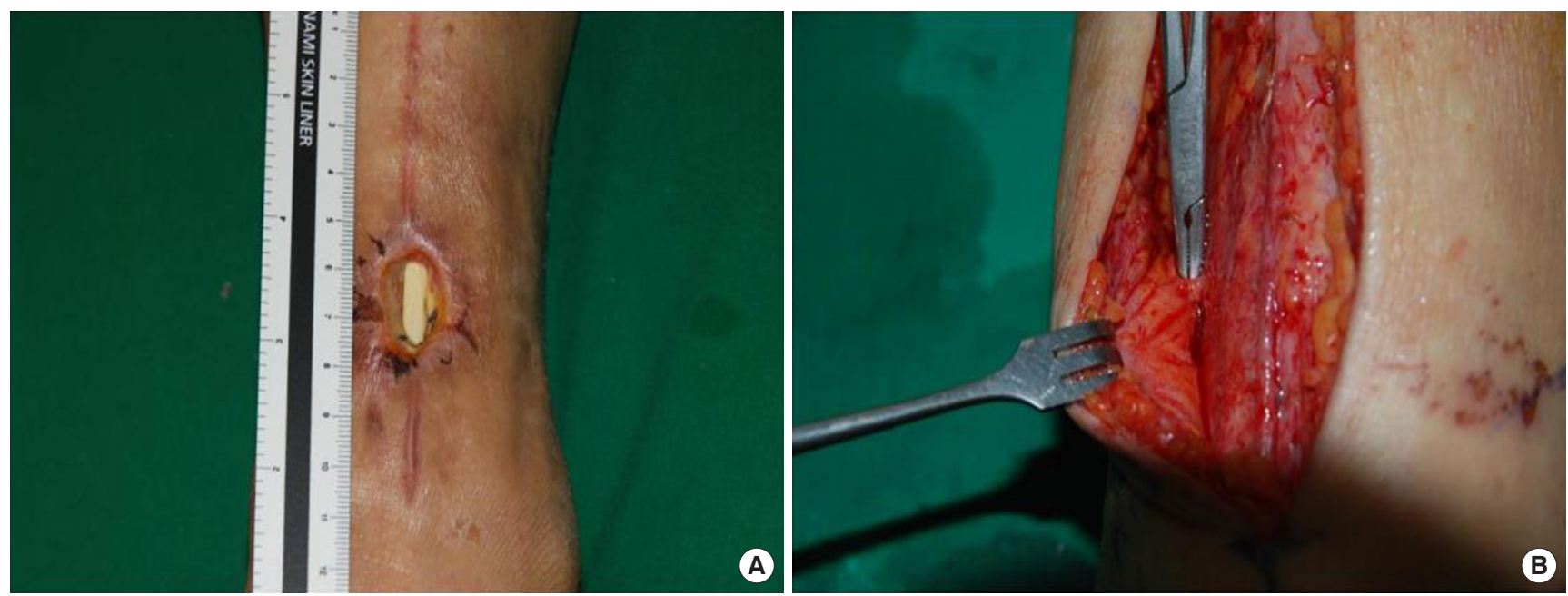

Fig. 1. (A) The preoperative finding of skin necrosis (size $2 \times 2 \mathrm{~cm}$ ) and exposed extensor tendon. (B) Exploratory incision, with identification and isolation of a posterior tibial artery perforator.
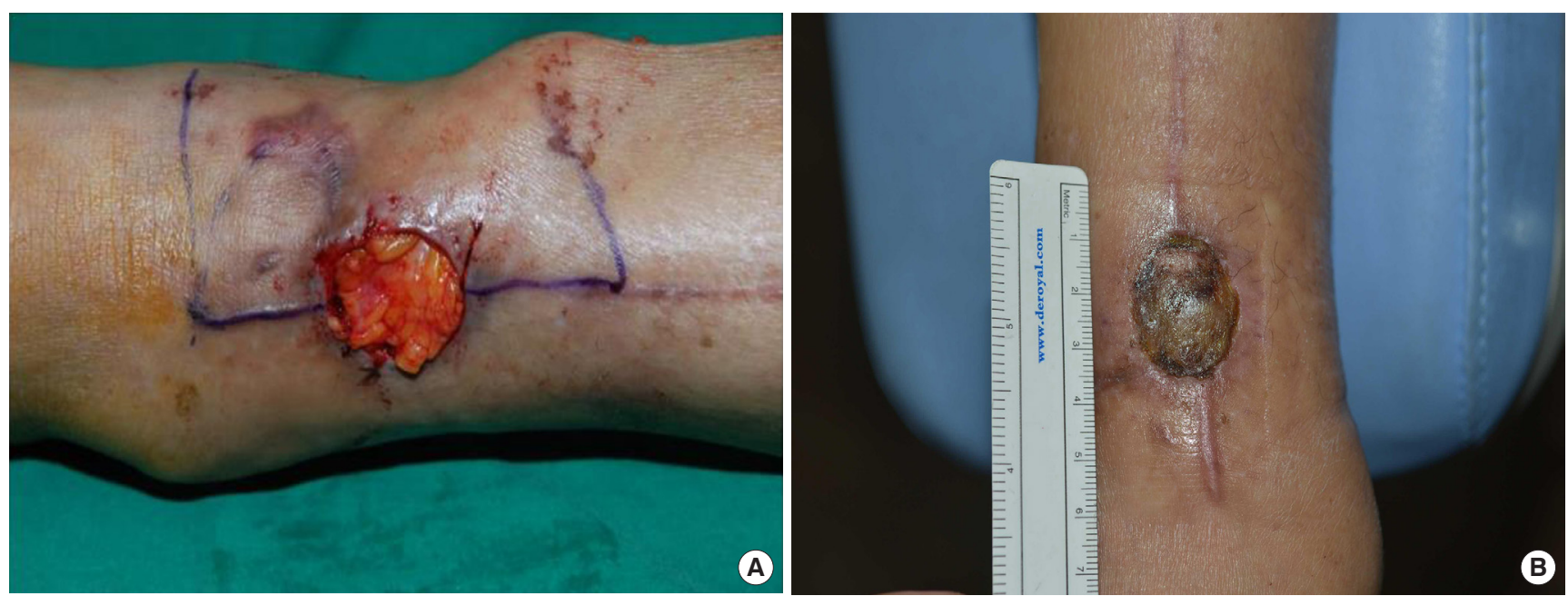

Fig. 2. (A) The flap was raised and transferred to the recipient area. (B) The reconstructed area showing a good contour and appearance at 3 months postoperatively. 

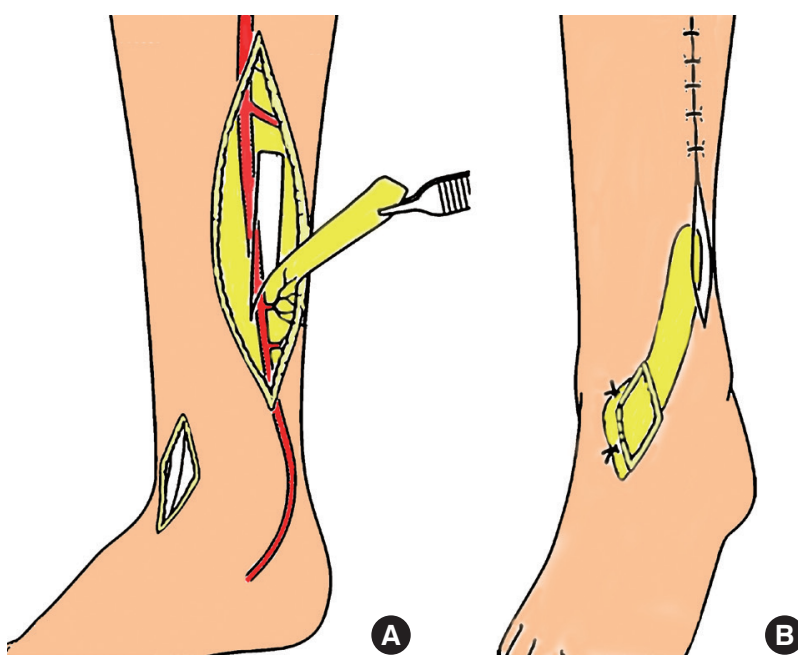

Fig. 3. Illustration of the operation. (A) The flap was elevated based on posterior tibial artery perforator. (B) Flap was transferred to the defect site.

is designed with respect to the defect and a limited anterior incision as for a fasciotomy is made through which the perforator is identified (Fig. 1B). The adipofascial flap can be reliably harvested and transferred to the defect. Split-thickness skin graft performed to cover the flap, and donor site is closed directly (Fig. 2A). After 3 months, flap and donor site remained well-healed state (Fig. 2B). Fig. 3 shows a schematic illustration of the operation.

\section{Discussion}

Soft tissue defects of the lower third of the leg present difficult reconstructive problems due to limited availability of local tissue, and donor site morbidity. Moreover, there are many cases that primary closure is impossible, even for small defects. In addition, local rotation, advancement, and transposition flaps are limited by the availableness of mobile skin. The free flap has improved the ability to cover soft tissue defects, however, the flap bulkiness that required for secondary procedures, and the risk of vascular failure are hesitant surgery [4]. Both muscle and fasciocutaneous flaps have their advantage and limitation for reconstruction of the leg. The muscle flap is suitable when you need to fill a lot of volumes. Due to using the vascularized tissue, muscle flaps may withstand contaminated or infected wounds. Fasciocutaneous flaps are indicated for the coverage of shallow wounds and can restore contour [2]. In the lower third of the leg, however, thin and pliable soft-tissue coverage is required to achieve a satisfac- tory aesthetic outcome and maintain the contour of the foot for shoe fitting $[2,5]$. The adipofascial flap, covered with a skin graft, can be one of the suitable methods of reconstruction that fulfills this important requirement.

Adipofascial flaps have inherent limitations, including flap thickness, hemorrhage or hematoma, difficulty in monitoring, and skin graft-related problems. The thinness of flaps makes them susceptible to pressure. The adipofascial flap has an affluent blood supply and forms a rich vascular network within the fascia. Therefore, the potential of intraoperative and postoperative bleeding are a reasonable concern. Another problem associated with adipofascial flaps is the possibility of the incomplete healing of the grafted skin [4]. In our case, a split-thickness skin graft was used, otherwise, other studies have reported that full-thickness skin graft minimizes the breakdown of the grafted skin [4].

The authors used a portable doppler to mark the perforator in the skin. However, since the doppler may be mistaken for the main blood flow as a perforator, it is used only for the reference to surgery. In fact, the perforator was confirmed with careful dissection after anterior incision.

In conclusion, the posterior tibial artery perforator-based adipofascial flap is can be one of the suitable methods for reconstruction of the defect of the distal third of leg and provides functional and aesthetic outcome with minimizing donor site morbidity.

\section{Conflict of interest}

No potential conflict of interest relevant to this article was reported.

\section{References}

1. Soltanian H, Garcia RM, Hollenbeck ST. Current Concepts in Lower Extremity Reconstruction. Plast Reconstr Surg 2015;136:815e-29e.

2. Schaverien MV, Hamilton SA, Fairburn N, et al. Lower limb reconstruction using the islanded posterior tibial artery perforator flap. Plast Reconstr Surg 2010;125:1735-43.

3. Lu TC, Lin CH, Lin CH, et al. The versatility of the pedicled peroneal artery perforator flaps for soft-tissue coverage of the lower leg and foot defects. J Plast Reconstr Aesthet Surg 2011;64:386-93.

4. Chung MS, Baek GH, Gong HS, et al. Lateral calcaneal artery adipofascial flap for reconstruction of the posterior heel 
of the foot. Clin Orthop Surg 2009;1:1-5.

5. Suliman MT. Distally based adipofascial flaps for dorsal foot and ankle soft tissue defects. J Foot Ankle Surg 2007;46: 464-9. 